\title{
New spectroscopic binaries among classical Cepheids. II.
}

\author{
L. Szabados ${ }^{1}$ and F. Pont ${ }^{2}$ \\ 1 Konkoly Observatory, Budapest XII, P.O. Box 67, H-1525 Budapest, Hungary \\ e-mail: szabados@buda.konkoly.hu \\ 2 Observatoire de Genève, 51 chemin des Maillettes, 1290 Sauverny, Switzerland
}

Received April 7; accepted May 11, 1998

\begin{abstract}
Radial velocity measurements of classical Cepheids obtained by the CORAVEL and ELODIE spectrographs have been analysed. The comparison with earlier radial velocity data resulted in the discovery of eight new spectroscopic binaries (in the $9.7-12^{\mathrm{m}} \cdot 4$ interval of mean $V$-brightness). An updated value of the pulsation period is also determined for the new SB-Cepheids.
\end{abstract}

Key words: Cepheids — binaries: spectroscopic

\section{Introduction}

Cepheid variables have been targets of numerous extensive (multicolour) photometric studies in view of their key-rôle in determining stellar structure and evolution as well as the cosmic distance scale through the regular pulsation of these variable stars. Unlike photometric data, there are only few projects aimed at measuring radial velocities of large numbers of Cepheids because such projects are more time-consuming than the photometric ones.

Radial velocity data are, however, indispensable in several respects. Their traditional use is for deriving radius variations via the Baade-Wesselink method from which the stellar radius can be derived as well as the luminosity and distance of the individual Cepheids (Gieren et al. 1993). With the advent of sensitive and precise radial velocity spectrometers (e.g. CORAVEL, ELODIE - Baranne et al. 1979, 1996), radial velocity of faint Cepheids can also be studied, allowing the analysis of Galactic kinematics and rotation parameters (Pont et al. 1997). The third major field of research where radial velocity data offer a significant contribution is the study of binary nature of Cepheids. Szabados (1995) pointed out the existence of a selection effect which hinders the discovery of duplicity among fainter Cepheids. As a matter of fact, the incidence of binaries among classical Cepheids exceeds 50 per cent.

Send offprint requests to: L. Szabados
Therefore, it is not surprising that new spectroscopic binaries involving a Cepheid component have been amply found from the data of extensive radial velocity projects. Such projects include: the observational campaigns of the Geneva group (Bersier et al. 1994; Pont et al. 1994, 1997) using the CORAVEL and more recently the ELODIE spectrographs, and those of the Moscow group (Gorynya et al. 1996 and the references therein), the latter group using a CORAVEL-type equipment. It is worth mentioning that spectroscopic binaries have been found even among Cepheids in the Magellanic Clouds (Imbert 1994), also with the help of the CORAVEL spectrograph.

The observational efforts of these teams have resulted in a unique data-base containing an unprecedented number of precise and homogeneous radial velocity measurements on classical Cepheids. The extended temporal coverage was a major contribution to help discover new SB-systems among Cepheids. Thus variations in the $\gamma$ velocity (i.e. the mean radial velocity averaged over a whole pulsational cycle) were reported e.g. in the case of BY Cas, AC Mon, UZ Sct, VY Sgr (Pont et al. 1994), MW Cyg (Gorynya et al. 1992b; Samus et al. 1993), and independently for BY Cas (Gorynya et al. 1994). Even the intercomparison of the data for Cepheids common in both samples allowed the discovery of SB-nature of such bright Cepheids as U Vul and SV Vul (Szabados 1996). (Later on, when supplementing with his own data, Imbert (1996) was able to determine the orbital elements for both the U Vul and MW Cyg systems.)

In addition to those recent datasets, there is a former project on Cepheid radial velocities performed as early as in the 1920-1930-es (Joy 1937). When comparing Joy's data with the recent ones, it turned out that many unrecognized SB-systems can be revealed even with the help of those historic data of limited accuracy (Szabados 1996).

The aim of this paper is similar: comparing the early radial velocity data with those reported by Pont et al. (1997). Because in their recent paper Pont et al. concentrated on the determination of the rotation of the Galactic 
disk, searching for effects of binary companions in the radial velocity data was not a main issue in that study. Nevertheless, using only their own high-precision data, CI Per is suspected to belong to a binary system. However, no attempt was made to compare the new data with the available previous radial velocity measurements. Their sample involved 48 remote, quite faint Cepheids. This is the reason why Pont et al.'s (1997) measurements form the first epoch radial velocity dataset for most of their programme stars. There have been, however, prior radial velocity data for 16 Cepheids in that sample - mostly obtained by Joy (1937) - allowing a comparison to be made between the mean values for the two epochs.

It turned out that eight Cepheids in that sample belong to spectroscopic binary systems unrecognized before. Following a brief description of the method of the analysis (Sect. 2), the available information on the new SBCepheids is published (Sect. 3), while Sect. 4 contains some concluding remarks.

\section{Method of the analysis}

When searching for changes in the $\gamma$-velocity caused by the orbital motion of the Cepheid around the common mass centre of the binary system, extreme care has to be taken not to mix spurious effects with the intrinsic radial velocity variation.

At first, the pulsational radial velocity curve has to be determined which necessitates knowledge of the pulsation period as accurately as possible. Use of inaccurate pulsation period causes a phase mismatch in the radial velocity curve which can give rise to increased scatter in the radial velocity curve. This, however, must not be interpreted as an orbital effect even though it appears as a vertical shift in the annual $\gamma$-velocity. Another negative consequence of the use of improper pulsation period is that it can smear any low amplitude orbital effect. The case of SV Vul clearly shows that even minor orbital $\gamma$-velocity variation can be detected if allowance is made for the continuously changing pulsation period (Szabados 1996).

If properly phased normal curves representing two different epochs are compared, only vertical shift can occur between the two curves, and this refers to the change in the mean radial velocity, i.e. to the orbital motion. This is, however, an idealistic case. In reality, the value of the radial velocity determined from the spectrum depends on both the circumstances in the stellar atmosphere having various impacts on the line profile (asymmetry, broadening, occasional emission - see e.g. Albrow \& Cottrell 1996; Butler 1993; Sabbey et al. 1995), and the method of determination of the radial velocity (Vinkó et al. 1998). Coupled with the problem of uncertainty in the early radial velocities (such as Joy's 1937, pioneering work), a reasonable lower limit of $\gamma$-velocity variation that can be attributed to the membership of the Cepheid in a binary system is four $\mathrm{km} \mathrm{s}^{-1}$ (Szabados 1996). Based on a homogeneous and precise dataset, this lower limit can be decreased considerably, see e.g. the case of SV Vul (Szabados 1996) again, and the remark on VW Pup, later on in this paper.

In order to determine the correct value of the pulsation period, the $\mathrm{O}-\mathrm{C}$-method was applied using the photometric data which are usually more accurate and available more frequently than radial velocity observations. The $\mathrm{O}-\mathrm{C}$-diagrams have been constructed for seven Cepheids in this sample (the only exception is V495 Mon). The commonly used method of $\mathrm{O}-\mathrm{C}$-diagram need not be introduced here, as to its details, the reader is referred to Willson (1986) (general information) and Szabados (1977) (application to Cepheids).

As to the other Cepheids for which the comparison of the recent radial velocity data with the first epoch values did not indicate noticeable change in the $\gamma$-velocity, the behaviour of the pulsation period was not studied during this project.

In all seven cases for which new pulsation period was determined, the new value only slightly differs from the catalogued period. The linear elements determined by the weighted least squares fit to the moments of the photometric normal maxima are indicated in the next section. Since no period change has been detected, nor assumed for the Cepheids under study, the $\mathrm{O}-\mathrm{C}$ graphs are not published here. Nevertheless, the normal maxima and the $\mathrm{O}-\mathrm{C}$-residuals utilized for the determination of the precise value of the pulsation period are given in tabular form (see Tables 2-8). These data, along with the bibliographic references may be useful for later studies and revisions of the pulsation period, keeping in mind that classical Cepheids undergo period changes of various origin (evolutionary, duplicity related, and erratic - see Szabados 1994).

The subsequent columns in the tables summarising the O-C-residuals contain the following data:

1. Moment of normal maximum; 2. Epoch as counted from the final ephemeris given among the remarks on individual variables in Sect. 3; 3. O-C-residual also calculated from the same ephemeris; 4 . Weight assigned to the given photometric series when performing the least squares fit for the period determination; 5. Type of the photometric data (vis: visual; pg: photographic; pe: photoelectric); 6 . Reference to the observational data.

In most cases, photographic and visual have been taken into account in order to incorporate those epochs when the first radial velocity series (Joy 1937) was obtained.

The list of the newly discovered SB-Cepheids is given in Table 1 which also contains the logarithm of the pulsation period (the precise value can be found as a remark at the respective Cepheid), the mean $V$-brightness and the difference between the mean values of the radial velocity determined from Joy's (1937) and the recent data (absolute value in $\mathrm{km} \mathrm{s}^{-1}$ ). This latter difference gives a qualitative estimate for the orbital effect and it is by no 
Table 1. List of the newly discovered SB-Cepheids

\begin{tabular}{llrr}
\hline Cepheid & $\log P$ & $\langle V\rangle$ & $\Delta v_{\gamma}$ \\
\hline YZ Aur & 1.260 & 10.4 & 10.6 \\
AS Aur & 0.502 & 11.9 & 8.2 \\
AA Gem & 1.053 & 9.7 & 14.2 \\
TX Mon & 0.940 & 11.0 & 7.0 \\
V495 Mon & 0.612 & 12.4 & $?$ \\
CS Ori & 0.590 & 11.4 & 26.7 \\
UX Per & 0.660 & 11.6 & 14.6 \\
VW Pup & 0.632 & 11.4 & 29.4 \\
\hline
\end{tabular}

means the amplitude of the orbital radial velocity variation. Because of the limited number of Joy's data, the arithmetic average of the radial velocities is not strictly equal to the $\gamma$-velocity but it serves as an approximation (see Pont et al. 1994b on the goodness of $\gamma$-velocity determinations from a few data points). For V495 Mon the available data are far too small to estimate the orbital effect but in view of the homogeneity of the data-set the variation in the $\gamma$-velocity is probably real.

The Cepheids involved in this sample can be commonly characterised as neglected from an observational point of view but fortunately the distribution of the available data allows the precise determination of the pulsation period. The much less numerous radial velocity data are only sufficient for revealing the variability in the $\gamma$-velocity, the orbital elements can be determined if more radial velocity data are available.

\section{Remarks on the individual Cepheids}

\section{YZ Aurigae}

The presence of a photometric companion has already been suspected by Madore (1977) and Madore \& Fernie (1980). The additional light from a blue companion star has been confirmed by Szabados (1998) based on the wavelength dependence of the photometric amplitudes in $U, B$, $V$ and $R$ bands.

The radial velocity of YZ Aur was measured at two epochs (Joy 1937, and present paper) separated by an interval longer than 20000 days. (In addition, there are two recent data published by Gorynya et al. 1992a). Although the descending branch of the radial velocity phase curve is not covered by recent data (see Fig. 1), the systematic shift in the $\gamma$-velocity is obvious between the two data series.

The new value of the pulsation period has been determined from the data collected in Table 2. The revised elements for the normal maxima are as follows:

$$
\begin{gathered}
C=\mathrm{JD} 2443816.417+18^{\mathrm{d}} 192830 \times E \\
\pm .030 \quad \pm .000043 .
\end{gathered}
$$

The radial velocity phase curve is shown plotted in Fig. 1 using this revised value of the pulsation period.
Table 2. $\mathrm{O}-\mathrm{C}$ residuals for $\mathrm{YZ}$ Aur

\begin{tabular}{crrrll}
\hline $\begin{array}{c}\text { JD } \\
2400000+\end{array}$ & $E$ & \multicolumn{1}{c}{ O-C w type Reference } \\
{$[\mathrm{d}]$}
\end{tabular}

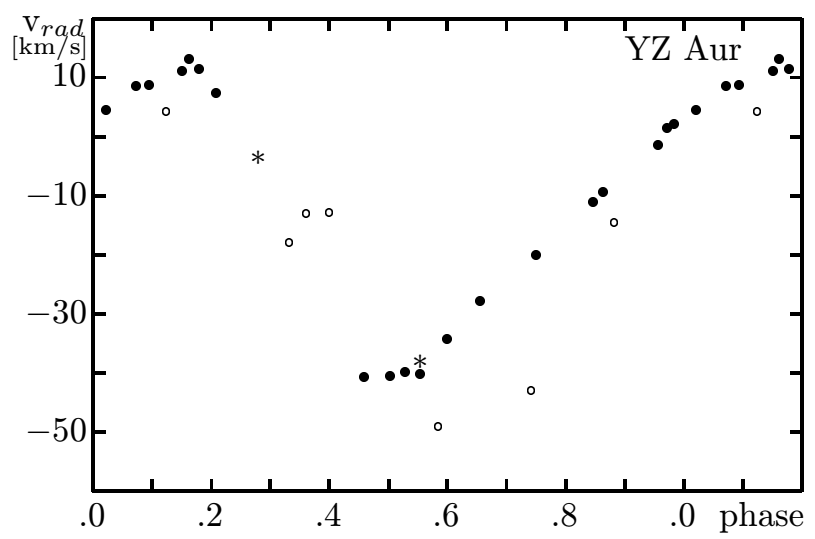

Fig. 1. Radial velocity curve of YZ Aur using the revised pulsation period of 18.192830 days. Zero phase was arbitrarily chosen at JD 2400000 for each radial velocity phase curve presented in this paper. Open circles denote Joy's (1937) data, asterisks mean Gorynya et al.'s (1992a) observations, while filled circles are used for showing data listed in the present paper

\section{AS Aurigae}

As to the photometric data, this Cepheid has been very much neglected (see Table 3 ) but fortunately there are two radial velocity measurement series widely separated in time (Joy 1937, and present paper) showing discordant $\gamma$-velocity values (see Fig. 2). Because the photometric observational material also spans a time interval longer than half a century, the phase matching can be considered as reliable when using the revised pulsation period. The scanty photometric data led to the following elements for the moments of brightness maxima:

$$
\begin{gathered}
C=\mathrm{JD} 2447648.646+3 \mathrm{~d} 175001 \times E \\
\pm .007 \quad \pm .000002 .
\end{gathered}
$$

\section{AA Geminorum}

Being the brightest Cepheid in this sample, AA Gem has 
Table 3. O-C residuals for AS Aur

\begin{tabular}{crrll}
\hline JD & $E$ & O-C w type Reference \\
$2400000+$ & {$[\mathrm{d}]$} & & \\
\hline 26325.338 & -6716 & -0.001 & 1 & vis Tsessevich (1952) \\
47648.634 & 0 & -0.012 & 3 & CCD Henden (1996) \\
48588.463 & 296 & 0.017 & 2 & CCD Schmidt \& Reiswig (1993) \\
\hline
\end{tabular}

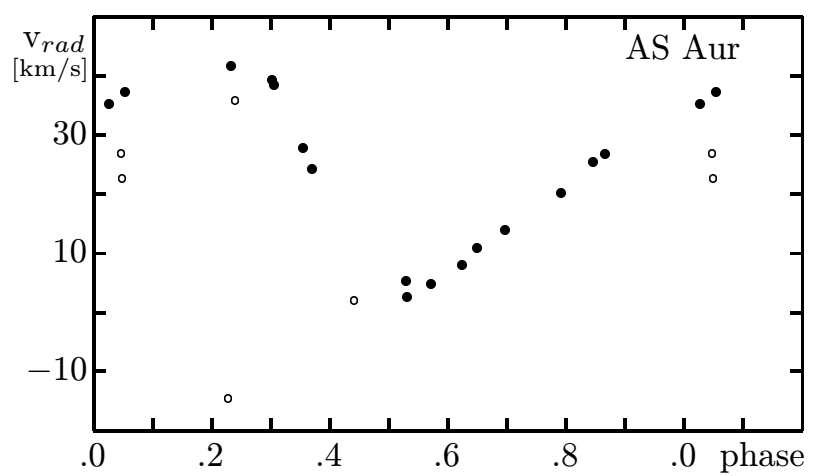

Fig. 2. Radial velocity curve of AS Aur folded on the revised pulsation period of 3.175001 days. Open circles: Joy's (1937) data, filled circles: present paper

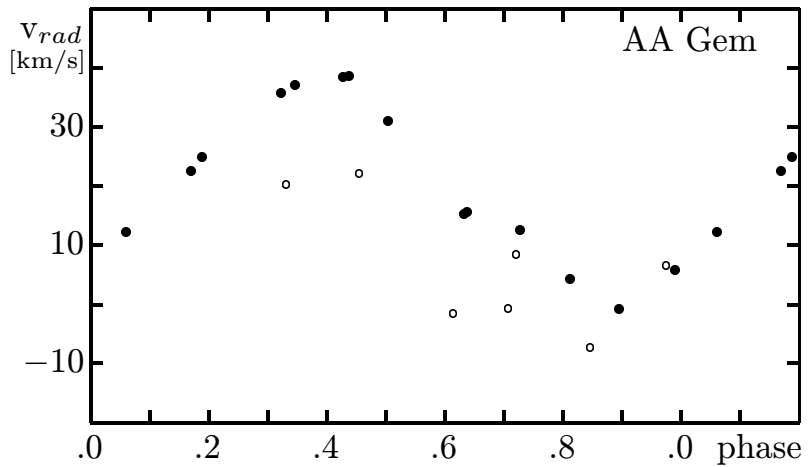

Fig. 3. Radial velocity curve of AA Gem using the revised pulsation period of 11.302450 days. Symbols are the same as in Fig. 2

a long record of photometric observations (see Table 4), therefore the new ephemeris is very reliable:

$$
\begin{gathered}
C=\mathrm{JD} 2443737.759+1 \mathrm{1}^{\mathrm{d}} 302450 \times E \\
\pm .030 \quad \pm .000036 .
\end{gathered}
$$

The photometric duplicity indicators led to controversial results: Madore's (1977) loop-method does not indicate a (blue) companion while the phase-method (Madore \& Fernie 1980) refers to a photometric companion. In their recent study, Evans \& Udalski (1994) conclude that the two faint companions are only optical, i.e. physically unrelated to AA Gem. The available two radial velocity datasets, however, result in significantly discordant $\gamma$ velocities (see Fig. 3): Joy's (1937) velocity data fall below the recent data by more than $10 \mathrm{~km} \mathrm{~s}^{-1}$ on the average.

\begin{tabular}{|c|c|c|c|}
\hline $\begin{array}{c}\text { JD } \\
2400000+\end{array}$ & $E$ & $\begin{array}{l}\text { O-C w type } \\
\text { [d] }\end{array}$ & Reference \\
\hline 25133.785 & -1646 & $-0.1411 \mathrm{pg}$ & Prager (1929) \\
\hline 25348.970 & -1627 & 0.2971 vis & Kukarkin (1940) \\
\hline 26015.515 & -1568 & -0.0021 vis & Beyer (1934) \\
\hline 27055.602 & -1476 & 0.2591 vis & Beyer (1934) \\
\hline 28061.251 & -1387 & -0.0101 vis & Martynov (1951) \\
\hline 28683.197 & -1332 & 0.3011 vis & Martynov (1951) \\
\hline 28953.877 & -1308 & $-0.2771 \mathrm{pg}$ & Koshkina (1963) \\
\hline 29157.579 & -1290 & $-0.0201 \mathrm{pg}$ & Chudovicheva (1952) \\
\hline 29439.991 & -1265 & -0.1691 vis & Martynov (1951) \\
\hline 29530.500 & -1257 & $-0.0791 \mathrm{pg}$ & Koshkina (1963) \\
\hline 30118.358 & -1205 & 0.0511 vis & Martynov (1951) \\
\hline 30785.592 & -1146 & 0.4411 vis & Martynov (1951) \\
\hline 31135.468 & -1115 & $-0.0591 \mathrm{pg}$ & Chudovicheva (1952) \\
\hline 32446.439 & -999 & $-0.1721 \mathrm{pg}$ & Chudovicheva (1952) \\
\hline 33543.332 & -902 & $0.3831 \mathrm{pg}$ & Chudovicheva (1952) \\
\hline 33599.199 & -897 & $-0.2621 \mathrm{pg}$ & Satyvoldiev (1970) \\
\hline 33972.437 & -864 & $-0.0051 \mathrm{pg}$ & Rosino \& Nobili (1955) \\
\hline 34029.050 & -859 & $0.0961 \mathrm{pg}$ & Koshkina (1963) \\
\hline 34537.701 & -814 & $0.1361 \mathrm{pg}$ & Rosino \& Nobili (1955) \\
\hline 36504.096 & -640 & $-0.0951 \mathrm{pg}$ & Satyvoldiev (1970) \\
\hline 36831.705 & -611 & -0.2572 pe & Weaver et al. (1960) \\
\hline 37634.342 & -540 & -0.0942 pe & Mitchell et al. (1964) \\
\hline 38436.673 & -469 & $-0.2371 \mathrm{pg}$ & Satyvoldiev (1970) \\
\hline 39250.725 & -397 & 0.0392 pe & Takase (1969) \\
\hline 43737.679 & & -0.0802 pe & Szabados (1981) \\
\hline 44404.529 & 59 & $-0.0753 \mathrm{pe}$ & Moffett \& Barnes (1984) \\
\hline 44980.992 & 110 & -0.0373 pe & Moffett \& Barnes (1984) \\
\hline 45693.028 & 173 & -0.0553 pe & Berdnikov (1986) \\
\hline 47422.431 & 326 & 0.0733 pe & Berdnikov (1992a) \\
\hline 47987.378 & 376 & -0.1022 pe & Evans \& Udalski (1994) \\
\hline 48518.746 & 423 & 0.0513 pe & Berdnikov (1992b) \\
\hline 50010.958 & 555 & 0.3392 pe & Berdnikov et al. (1997) \\
\hline
\end{tabular}

Table 4. $\mathrm{O}-\mathrm{C}$ residuals for AA Gem

\section{TX Monocerotis}

Pel's (1978) multicolour photometry indicated the presence of a companion but no detailed spectroscopic study of this Cepheid has been performed yet. Vinkó (1991) searched for evidence of a companion in the $\mathrm{O}-\mathrm{C}$ diagram of TX Mon but the scatter due to inaccuracy of the early photometric data do not allow to reveal the lighttime effect, if any. He approximated the $\mathrm{O}-\mathrm{C}$ graph by a parabola assuming a continuous minor increase in the pulsation period. Here the pulsation period is assumed to be constant and the resulting linear ephemeris (determined from the data in Table 5)

$$
\begin{gathered}
C=\mathrm{JD} 2444982.854+8.701903 \times E \\
\pm .092 \quad \pm .000078 .
\end{gathered}
$$

is as acceptable as Vinkó's parabolic one.

The available radial velocity data, however, clearly show the effect of a physical companion (see Fig. 4). Not only are Joy's (1937) velocity values systematically smaller than the "normal" value of the corresponding phase of the 
Table 5. $\mathrm{O}-\mathrm{C}$ residuals for TX Mon

\begin{tabular}{|c|c|c|c|c|}
\hline $\begin{array}{c}\text { JD } \\
2400000+\end{array}$ & $E$ & $\begin{array}{c}\mathrm{O}-\mathrm{C} \\
{[\mathrm{d}]}\end{array}$ & w type & Reference \\
\hline 18909.53 & -2893 & 1.28 & $1 \mathrm{pg}$ & Fatkina (1950) \\
\hline 27048.309 & -2061 & 0.077 & 1 vis & Florya \& Kukarkina (195: \\
\hline 28596.57 & -1883 & -0.60 & $1 \mathrm{pg}$ & Fatkina (1950) \\
\hline 29283.59 & -1804 & -1.03 & $1 \mathrm{pg}$ & Fatkina (1950) \\
\hline 29719.899 & -1754 & 0.184 & $1 \mathrm{pg}$ & Erleksova (1960) \\
\hline 30641.927 & -1648 & -0.191 & $1 \mathrm{pg}$ & Erleksova (1960) \\
\hline 32973.997 & -1380 & -0.231 & $1 \mathrm{pg}$ & Erleksova (1960) \\
\hline 33600.434 & -1308 & -0.331 & $1 \mathrm{pg}$ & Erleksova (1960) \\
\hline 35549.796 & -1084 & -0.195 & $1 \mathrm{pg}$ & Erleksova (1960) \\
\hline 35558.914 & -1083 & 0.221 & 3 pe & Walraven et al. (1958) \\
\hline 36246.019 & -1004 & -0.124 & $1 \mathrm{pg}$ & Erleksova (1960) \\
\hline 36837.767 & -936 & -0.106 & 3 pe & Mitchell et al. (1964) \\
\hline 40832.092 & -477 & 0.046 & 3 pe & Pel (1976) \\
\hline 44982.890 & 0 & 0.036 & 3 pe & Moffett \& Barnes (1984) \\
\hline 48515.927 & 406 & 0.100 & 3 pe & Berdnikov (1992b) \\
\hline 49812.500 & 555 & 0.090 & 3 pe & Berdnikov \& Turner (1995) \\
\hline
\end{tabular}

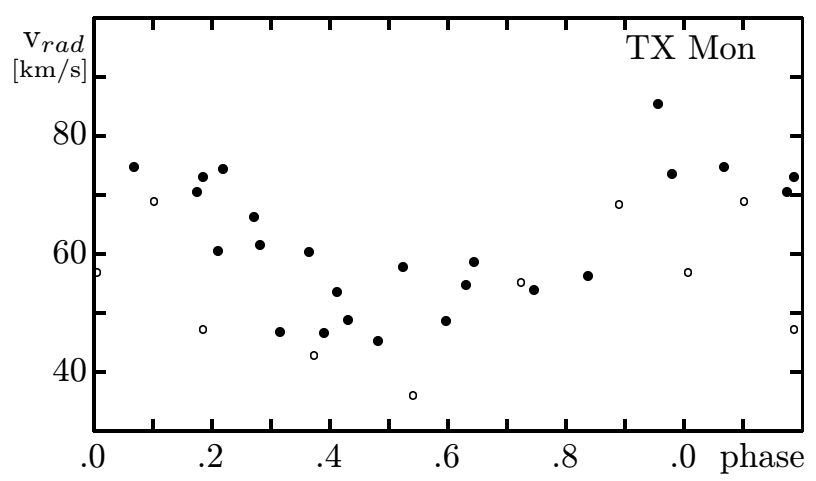

Fig. 4. Radial velocity curve of TX Mon folded on the revised pulsation period of 8.701903 days. Symbols are the same as in Fig. 2

second-epoch radial velocity curve but the recent highprecision data collected during a time interval as short as four years show a wide scatter, indicating variations in the $\gamma$-velocity due to orbital motion. The number of the available data points justified that a search for periodicity be performed, in order to obtain a preliminary value of the orbital period. A Lafler-Kinman-type algorithm (Lafler \& Kinman 1965) indicates that the orbital period is near 470 days (the uncertainty being as large as \pm 30 days), and longer periods can be excluded. This means that TX Mon is a classical Cepheid with one of the shortest known orbital period. A Fourier-type algorithm (Deeming 1975), however, could not confirm this preliminary value but no other value for the orbital periodicity could be guessed.

\section{V495 Monocerotis}

The faintest Cepheid in this sample has only a brief history of observations. Even the pulsation period could not be improved based on the available photometric data cov-

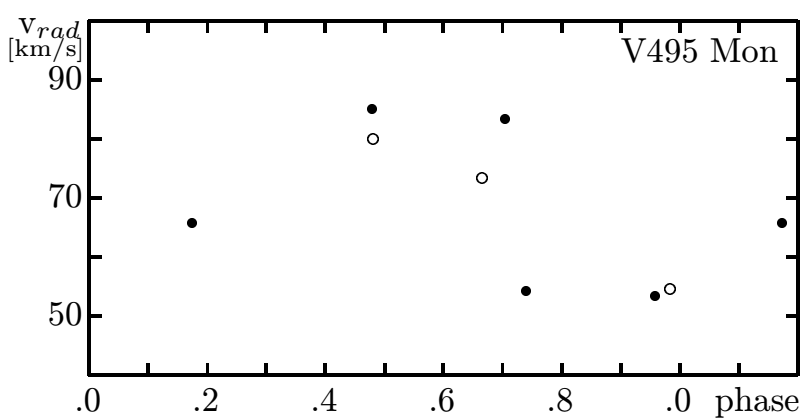

Fig. 5. Radial velocity curve of V495 Mon folded on the pulsation period of 4.096583 days. All observations were obtained by Pont et al. (1997). Filled circles denote radial velocity data taken in Nov. 1993/Mar. 1994, open circles are the data from Dec. 1994-Jan. 1995

ering only a decade, so the value of 4.096583 days, published in the GCVS, has been used.

The radial velocity data obtained by the ELODIEspectrograph (Pont et al. 1997) are plotted in Fig. 5. It is clearly seen that the data show an annual shift, indicative of the orbital motion of the Cepheid around the mass centre of a binary system. Since these data were acquired within two consecutive observational seasons, any subtle error in the pulsation period cannot modify this conclusion. Duplicity of V495 Mon, however, has to be confirmed by further radial velocity data because the present data have been obtained at the brightness limit for the ELODIE, and the correlation functions sometimes were not very clean.

\section{CS Orionis}

The updated ephemeris determined from the data listed in Table 6 is as follows:

$$
\begin{gathered}
C=\mathrm{JD} 2443609.046+3.889281 \times E \\
\pm .039 \quad \pm .000014 .
\end{gathered}
$$

The photometric amplitudes in $U, B, V$, and $R$ bands indicate the presence of a blue companion (Szabados 1998).

This companion may be responsible for the orbital effect detectable in the radial velocity data (see Fig. 6). The difference between the mean velocity averaged over one pulsational cycle exceeds $20 \mathrm{~km} \mathrm{~s}^{-1}$, if Joy's (1937) and the recent data are compared.

\section{UX Persei}

The revised ephemeris determined from the data appearing in Table 7 is as follows:

$$
\begin{gathered}
C=\mathrm{JD} 2448981.686+4.565733 \times E \\
\pm .027 \quad \pm .000011 .
\end{gathered}
$$

Although Madore's (1977) loop-method, based on the $U-$ $B$ and $B-V$ colour indices, does not indicate the presence of a secondary star, UX Per seems to have a photometric companion based on the phase-method (Madore \& Fernie 1980). 
Table 6. $\mathrm{O}-\mathrm{C}$ residuals for $\mathrm{CS}$ Ori

\begin{tabular}{|c|c|c|c|}
\hline $\begin{array}{c}\text { JD } \\
2400000+\end{array}$ & $\begin{array}{c}\mathrm{O}-\mathrm{Cu} \\
{[\mathrm{d}]}\end{array}$ & $\mathrm{v}$ type & Reference \\
\hline 16099.830 & $-7073-0.3311$ & $\mathrm{pg}$ & Parenago (1933) \\
\hline 22299.689 & 0.0141 & $\mathrm{pg}$ & Parenago (1933) \\
\hline 29281.125 & 0.1901 & $\mathrm{pg}$ & Kukarkina (1954) \\
\hline 33948.296 & 0.2241 & $\mathrm{pg}$ & Kukarkina (1954) \\
\hline 37499.083 & 0.0972 & pe & Mitchell et al. (1964) \\
\hline 43609.113 & 0.0673 & pe & Henden (1980) \\
\hline 48672.803 & $1302-0.0873$ & CCL & Schmidt et al. (1995) \\
\hline 49800.706 & $1592-0.0753$ & pe & Pont et al. (1997) \\
\hline
\end{tabular}

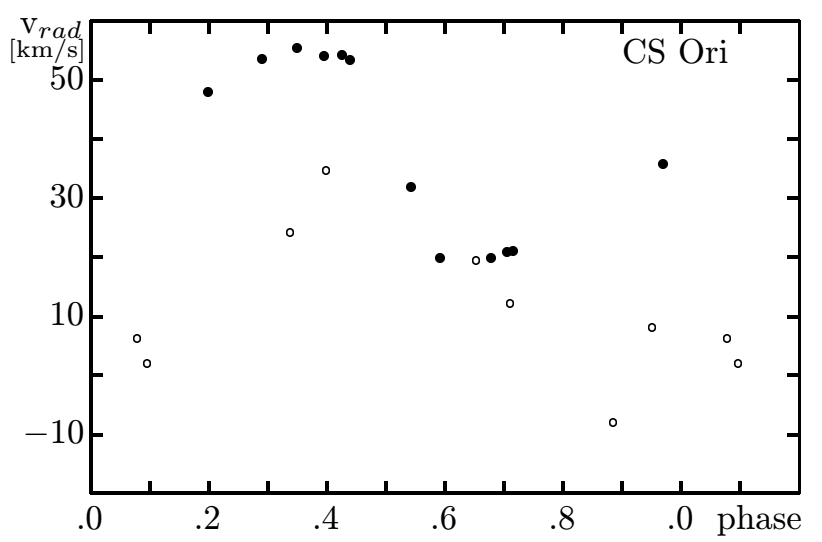

Fig. 6. Radial velocity curve of CS Ori folded on the revised pulsation period of 3.889281 days. Symbols are the same as in Fig. 2

Table 7. O-C residuals for UX Per

\begin{tabular}{crrrll}
\hline JD & $E$ & $\begin{array}{c}\text { O-C w } \\
{[\mathrm{d}]}\end{array}$ & & \\
$2400000+$ & \multicolumn{1}{c}{ type Reference } \\
\hline 26449.789 & -4935 & -0.005 & 1 & vis & Kukarkin (1932) \\
29280.471 & -4315 & -0.077 & 1 & pg & Lesyunina (1963) \\
34695.747 & -3129 & 0.240 & 1 & pg & Lesyunina (1963) \\
36882.452 & -2650 & -0.042 & 3 & pe & Bahner et al. (1962) \\
37631.265 & -2486 & -0.009 & 3 & pe & Mitchell et al. (1964) \\
49981.675 & 0 & -0.011 & 3 & CCD & Schmidt \& Seth (1996) \\
49990.720 & 221 & 0.008 & 3 & pe & Berdnikov et al. (1997) \\
\hline
\end{tabular}

The radial velocity data clearly indicate a spectroscopic companion (see Fig. 7): Joy's (1937) data are systematically less negative (but a single point) than the CORAVEL-measurements, the difference being significant, about $15 \mathrm{~km} \mathrm{~s}^{-1}$.

\section{VW Puppis}

The revised ephemeris based on the photometric data (see Table 8) is quite precise:

$$
\begin{gathered}
C=\mathrm{JD} 2443581.241+4.285298 \times E \\
\pm .014 \quad \pm .000009 .
\end{gathered}
$$

The variation in the $\gamma$-velocity caused by the orbital motion is the largest in this sample (see Fig. 8): the difference

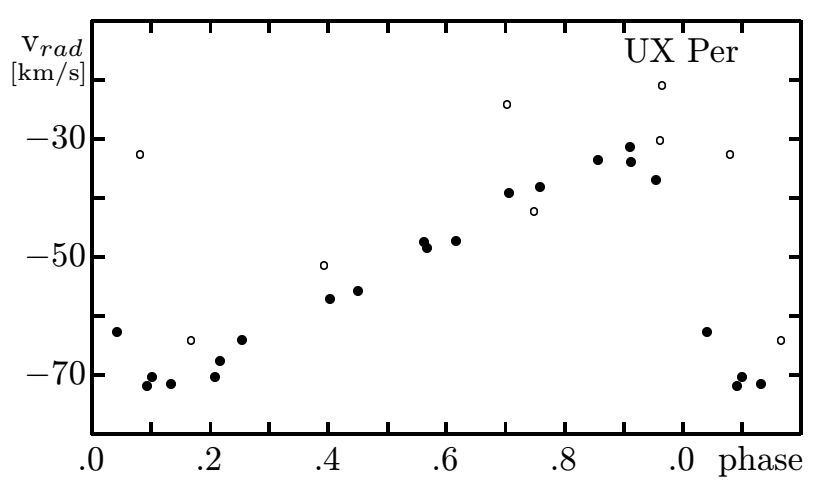

Fig. 7. Radial velocity curve of UX Per folded on the revised pulsation period of 4.565733 days. Symbols are the same as in Fig. 2

Table 8. O-C residuals for VW Pup

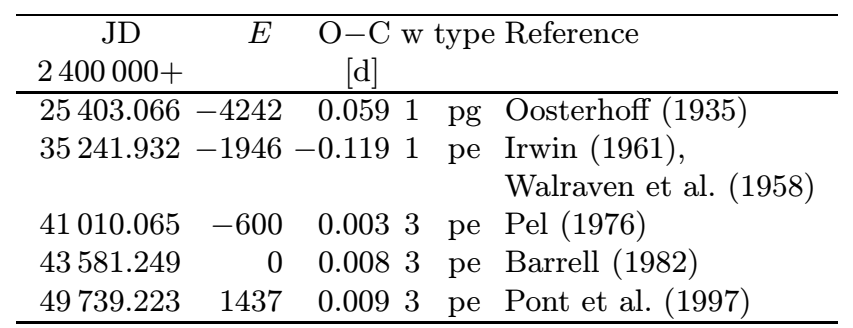

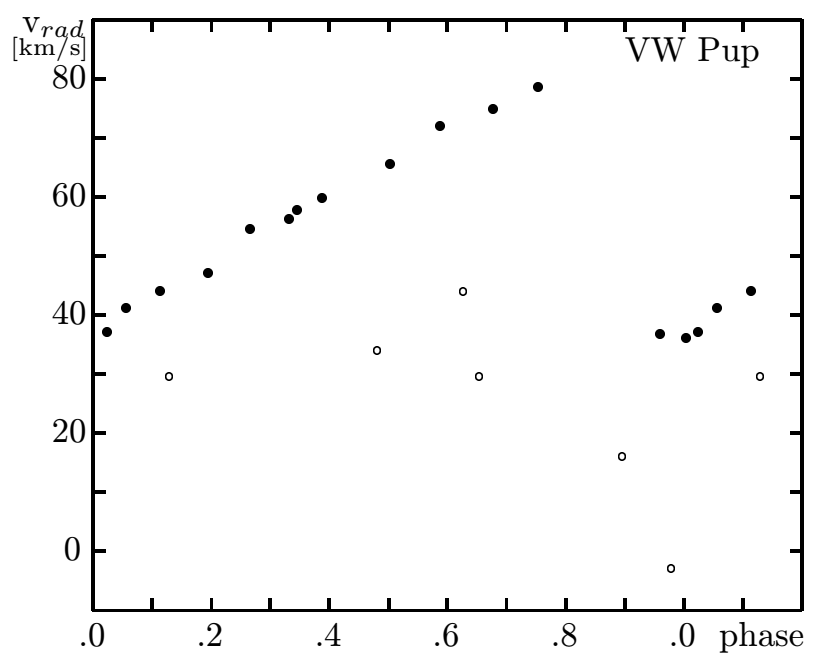

Fig. 8. Radial velocity curve of VW Pup folded on the revised pulsation period of 4.285298 days. Symbols are the same as in Fig. 2

between Joy's (1937) and the CORAVEL-data of the corresponding phase is about $25 \mathrm{~km} \mathrm{~s}^{-1}$. A closer inspection of the CORAVEL-data reveals that even an annual shift is noticeable: an increase of about $1 \mathrm{~km} \mathrm{~s}^{-1}$ in the $\gamma$-velocity is observed in this homogeneous dataset. 
Table 9. Radial velocity data for the programme stars (for V495 Mon: ELODIE-data, for the other seven Cepheids: CORAVELdata)

\begin{tabular}{|c|c|c|c|c|c|c|c|c|}
\hline JD $2400000+$ & $\begin{array}{c}v_{\mathrm{rad}} \\
{\left[\mathrm{km} \mathrm{s}^{-1}\right]}\end{array}$ & $\begin{array}{c}\sigma \\
{\left[\mathrm{km} \mathrm{s}^{-1}\right]}\end{array}$ & JD $2400000+$ & $\begin{array}{c}v_{\mathrm{rad}} \\
{\left[\mathrm{km} \mathrm{s}^{-1}\right]}\end{array}$ & $\begin{array}{c}\sigma \\
{\left[\mathrm{km} \mathrm{s}^{-1}\right]}\end{array}$ & JD $2400000+$ & $\begin{array}{c}v_{\mathrm{rad}} \\
{\left[\mathrm{km} \mathrm{s}^{-1}\right]}\end{array}$ & $\begin{array}{c}\sigma \\
{\left[\mathrm{km} \mathrm{s}^{-1}\right.}\end{array}$ \\
\hline$Y Z A u r$ & & & $A A$ Gem & & & CS Ori & & \\
\hline 49639.637 & -39.81 & 0.61 & 49656.600 & 38.70 & 0.49 & 49681.555 & 35.87 & 0.42 \\
\hline 49656.571 & -40.69 & 0.85 & 49663.638 & 12.28 & 0.39 & 49684.419 & 20.96 & 0.66 \\
\hline 49663.626 & -11.09 & 0.58 & 49681.426 & 15.22 & 0.38 & 49749.501 & 53.50 & 0.68 \\
\hline 49684.321 & 2.06 & 0.55 & 49682.485 & 12.58 & 0.39 & 49768.369 & 53.53 & 0.53 \\
\hline 49687.558 & 13.12 & 0.77 & 49684.375 & -0.79 & 0.40 & 49769.346 & 31.88 & 0.69 \\
\hline 49768.293 & -34.19 & 0.53 & 49721.602 & 24.89 & 0.46 & 49776.374 & 55.36 & 0.56 \\
\hline 49769.299 & -27.73 & 0.60 & 49768.328 & 35.83 & 0.39 & 49777.319 & 19.87 & 1.03 \\
\hline 49777.299 & 8.76 & 0.76 & 49770.381 & 31.09 & 0.49 & 50437.730 & 54.12 & 0.66 \\
\hline 49778.321 & 11.11 & 0.97 & 50438.744 & 15.68 & 0.46 & 50441.744 & 54.31 & 0.49 \\
\hline 49779.354 & 7.42 & 0.91 & 50440.694 & 4.27 & 0.44 & 50442.720 & 19.82 & 0.68 \\
\hline 50130.388 & -40.51 & 0.70 & 50442.707 & 5.85 & 0.39 & 50444.745 & 48.01 & 0.77 \\
\hline 50131.309 & -40.09 & 0.87 & 50444.739 & 22.54 & 0.46 & 50446.754 & 21.07 & 0.68 \\
\hline 50393.635 & 1.52 & 0.69 & 50446.741 & 37.10 & 0.43 & & & \\
\hline 50394.530 & 4.58 & 0.60 & 50447.665 & 38.54 & 0.51 & $U X P e r$ & & \\
\hline 50462.342 & -20.01 & 0.43 & & & & & & \\
\hline 50464.411 & -9.32 & 0.58 & TX Mon & & & 49364.296 & -31.32 & 0.73 \\
\hline 50484.315 & -1.47 & 0.61 & & & & 49365.312 & -71.48 & 0.88 \\
\hline 50486.410 & 8.61 & 0.70 & 49364.473 & 56.24 & 0.57 & 49367.299 & -48.47 & 0.56 \\
\hline \multirow[t]{2}{*}{50488.362} & 11.47 & 0.80 & 49366.487 & 74.66 & 0.48 & 49374.255 & -71.80 & 1.32 \\
\hline & & & 49422.298 & 45.25 & 0.60 & 49419.282 & -36.92 & 1.52 \\
\hline \multirow[t]{2}{*}{$A S$ Aur } & & & 49423.292 & 48.65 & 0.66 & 49422.308 & -47.36 & 0.82 \\
\hline & & & 49639.686 & 66.79 & 0.49 & 49639.598 & -70.32 & 0.77 \\
\hline 49639.649 & 2.70 & 0.87 & 49656.623 & 66.35 & 0.51 & 49655.571 & -39.13 & 0.75 \\
\hline 49656.591 & 26.89 & 0.58 & 49663.670 & 74.28 & 0.74 & 49656.512 & -33.85 & 0.76 \\
\hline 49681.454 & 13.90 & 0.54 & 49681.520 & 66.26 & 0.46 & 49663.531 & -55.75 & 0.74 \\
\hline 49682.500 & 35.39 & 0.50 & 49769.360 & 60.28 & 0.54 & 49812.338 & -62.73 & 1.08 \\
\hline 49753.394 & 27.81 & 0.75 & 49777.335 & 61.53 & 0.73 & 49813.307 & -64.03 & 0.97 \\
\hline 49756.410 & 38.47 & 0.76 & 49809.311 & 85.39 & 0.58 & 50394.556 & -47.42 & 0.42 \\
\hline 49768.309 & 37.33 & 0.58 & 50098.464 & 73.07 & 0.69 & 50462.316 & -57.12 & 0.60 \\
\hline 49769.311 & 24.22 & 0.80 & 50100.445 & 53.54 & 0.64 & 50464.392 & -33.60 & 0.83 \\
\hline 49779.341 & 5.37 & 1.06 & 50101.412 & 57.70 & 0.51 & 50484.294 & -67.58 & 0.85 \\
\hline 50395.597 & 8.08 & 0.77 & 50102.459 & 58.55 & 0.47 & 50488.337 & -70.33 & 1.18 \\
\hline 50462.356 & 10.91 & 0.78 & 50135.406 & 48.73 & 0.33 & 50491.335 & -38.20 & 0.66 \\
\hline 50464.422 & 39.39 & 0.80 & 50437.748 & 70.47 & 0.70 & & & \\
\hline 50484.330 & 4.79 & 0.71 & 50439.635 & 46.57 & 0.41 & $V W P u p$ & & \\
\hline 50486.428 & 41.71 & 0.73 & 50441.713 & 54.68 & 0.47 & & & \\
\hline 50488.376 & 25.59 & 0.60 & 50442.712 & 53.82 & 0.43 & 49656.650 & 74.90 & 0.71 \\
\hline \multirow[t]{14}{*}{50491.378} & 20.30 & 0.59 & 50444.752 & 73.47 & 0.60 & 49681.617 & 65.63 & 0.60 \\
\hline & & & 50446.761 & 60.45 & 0.57 & 49684.582 & 47.21 & 0.46 \\
\hline & & & 50447.681 & 46.69 & 0.51 & 49768.396 & 78.62 & 0.62 \\
\hline & & & & & & 49796.587 & 56.21 & 0.53 \\
\hline & & & V495 Mon & & & 49799.552 & 37.13 & 0.93 \\
\hline & & & & & & 49812.315 & 36.11 & 0.69 \\
\hline & & & 49317.694 & 54.348 & 0.403 & 50437.783 & 36.90 & 0.68 \\
\hline & & & 49318.591 & 53.426 & 0.132 & 50439.619 & 59.76 & 0.39 \\
\hline & & & 49319.472 & 65.686 & 0.137 & 50442.727 & 44.08 & 0.59 \\
\hline & & & 49320.721 & 85.030 & 0.995 & 50443.720 & 57.84 & 0.53 \\
\hline & & & 49415.868 & 83.314 & 0.658 & 50444.757 & 71.94 & 0.62 \\
\hline & & & 49695.582 & 54.607 & 0.181 & 50446.767 & 41.22 & 0.58 \\
\hline & & & 49697.616 & 80.000 & 0.168 & & & \\
\hline & & & 49739.339 & 73.370 & 0.482 & & & \\
\hline
\end{tabular}




\section{Conclusion}

The discovery that eight more classical Cepheids have spectroscopic companion, also supports the facts (Szabados 1995) that the incidence of binaries among classical Cepheids is larger than 50 per cent, and there are lots of spectroscopic binaries among Cepheids fainter than 8th magnitude whose duplicity remains to be discovered.

The modern radial velocity spectrometers (CORAVEL, ELODIE, etc.) attached to a telescope of about $1 \mathrm{~m}$ diameter are capable of measuring stars of 12-13th magnitude within a reasonably short time, and the precision of the radial velocity determination allows the detection of very small orbital effects (less than one $\mathrm{km} \mathrm{s}^{-1}$ change in the $\gamma$-velocity), if homogeneous datasets covering several seasons are available. The shortest known orbital period for classical Cepheids is slightly longer than one year (Szabados 1992), while the other extremum, the longest still detectable orbital period is as long as several decades ( $\mathrm{T}$ Mon).

The earlier radial velocity data of limited precision are useful for revealing the orbital motion if there is only a short dataset of recent radial velocity values, which is the case for the stars discussed in the present paper. The next step, then, is the extensive study of these newly recognized spectroscopic binaries. For the time being, none of the orbital elements can be determined from the available data. There are, however, several pieces of information that can be deduced from the pattern of the $\gamma$-velocity change, such as the short orbital period of TX Mon and the several-year-long orbital period is adequate to the observations of VW Pup. All Cepheids whose binary nature is reported here show considerable amplitude of $\gamma$-velocity variation (only V495 Mon may be an exception). For the sake of information, the new radial velocity data on the eight new SB-Cepheids obtained by the CORAVEL and ELODIE spectrographs are listed in Table 9.

The detection of numerous spectroscopic binaries among Cepheids justifies that the time-consuming project of collecting radial velocity data of Cepheids is an observational programme worthy to be carried out because binary Cepheids are key-objects for both astrophysics (stellar evolution) and cosmology (distance scale).

Acknowledgements. This project has been partly financed by Hungarian OTKA-project T022946. L.Sz. is grateful to Dr. Mária Kun for her remarks contributing to the improvement of the manuscript.

\section{References}

Albrow M.D., Cottrell P.L., 1996, MNRAS 278, 337

Bahner K., Hiltner W.A., Kraft R.P., 1962, ApJS 6, 319

Baranne A., Mayor M., Poncet J.L., 1979, Vistas in Astr. 23, 279

Baranne A., Queloz D., Mayor M., et al., 1996, A\&A 315, 658

Barrell S.L., 1982, MNRAS 200, 139
Berdnikov L.N., 1986, Perem. Zv. 22, 369

Berdnikov L.N., 1992a, Astron. Astrophys. Trans. 2

Berdnikov L.N., 1992b, Pis'ma v AZh 18, No. 4, 325

Berdnikov L.N., Turner D.G., 1995, Pis'ma v AZh 21, 803

Berdnikov L.N., Ignatova V.V., Vozyakova O.V., 1997, Astron. Astrophys. Trans. 14, 237

Bersier D., Burki G., Mayor M., Duquennoy A., 1994, A\&AS 108, 25

Beyer M., 1930, Erg. Astron. Nachr. 8, No. 3

Beyer M., 1934, Astron. Nachr. 252, 85

Butler R.P., 1993, ApJ 415, 323

Chudovicheva O.N., 1952, Perem. Zv. 9, 134

Deeming T.J., 1975, Ap\&SS 36, 137

Detre L., 1935, Astr. Nachr. 257, 361

Erleksova G.E., 1960, Dushanbe Byull., No. 29, 23

Evans N.R., Udalski A., 1994, AJ 108, 653

Fatkina T.D., 1950, Perem. Zv. 7, 216

Filatov G.S., 1958, Perem. Zv. 12, 224

Florya N.F., Kukarkina N.P., 1953, Sternberg Tr. 23

Gieren W.P., Barnes T.G., Moffett T.J., 1993, ApJ 418, 135

Gorynya N.A., Irsmambetova T.R., Rastorgouev A.S., Samus N.N., 1992a, Pis'ma v AZh 18, No. 9, 777

Gorynya N.A., Samus N.N., Irsmambetova T.R., et al., 1992b, IBVS, No. 3776

Gorynya N.A., Samus N.N., Rastorgouev A.S., 1994, IBVS, No. 4130

Gorynya N.A., Samus N.N., Rastorgouev A.S., Sachkov M.E., 1996, Pis'ma v AZh 22, 198

Henden A.A., 1980, MNRAS 192, 621

Henden A.A., 1996, AJ 112, 2757

Imbert M., 1994, A\&AS 105, 1

Imbert M., 1996, A\&AS 116, 497

Irwin J.B., 1961, ApJS 6, 253

Joy A.H., 1937, ApJ 86, 363

Koshkina L.N., 1963, Perem. Zv. 14, 474

Kukarkin B.V., 1932, Perem. Zv. 4, No. 1, 1

Kukarkin B.V., 1940, Sternberg Tr. 13, No. 1, 118

Kukarkina N.P., 1954, Perem. Zv. 10, 98

Lafler J., Kinman T.D., 1965, ApJS 11, 216

Lesyunina N.S., 1963, Perem. Zv. 14, 351

Madore B.F., 1977, MNRAS 178, 505

Madore B.F., Fernie J.D., 1980, PASP 92, 315

Martynov D.Ya., 1951, Publ. Engelhardt Obs., No. 26, 3

Mitchell R.I., Iriarte B., Steinmetz D., Johnson H.L., 1964, Bol. Obs. Tonantzintla y Tacubaya 3, No. 24

Moffett T.J., Barnes T.G. III, 1984, ApJS 55, 389

Oosterhoff P.Th., 1935, Harvard Bul., No. 900, 3

Parenago P.P., 1933, Perem. Zv. 4, 145

Pel J.W., 1976, A\&AS 24, 413

Pel J.W., 1978, A\&A 62, 75

Pont F., Burki G., Mayor M., 1994a, A\&AS 105, 165

Pont F., Mayor M., Burki G., 1994b, A\&A 285, 415

Pont F., Queloz D., Bratschi P., Mayor M., 1997, A\&A 318, 416

Prager R., 1929, Kl. Veröff. Berlin-Babelsberg, No. 6

Rosino L., Nobili F., 1955, Bologna Pubbl. 6, No. 15

Sabbey C.N., Sasselov D.D., Fieldus M.S., Lester J.B., Venn K.A., Butler R.P., 1995, ApJ 446, 250

Samus N.N., Gorynya N.A., Kulagin Yu.V., Rastorgouev A.S., 1993, IBVS, No. 3934

Satyvoldiev V., 1970, Dushanbe Byull., No. 54, 21 
Schmidt E.G., Reiswig D.E., 1993, AJ 106, 2429

Schmidt E.G., Seth A., 1996, AJ 112, 2769

Schmidt E.G., Chab J.R., Reiswig D.E., 1995, AJ 109, 1239

Szabados L., 1977, Mitt Sternw ung. Akad. Wiss., Budapest, No. 70

Szabados L., 1981, Commun. Konkoly Obs. Hung. Acad. Sci., Budapest, No. 77

Szabados L., 1992, in: Complementary Approaches to Double and Multiple Star Research, Proc. IAU. Coll., No. 135, McAlister H.A. and Hartkopf W.I. (eds.), ASP Conf. Ser. 32,358

Szabados L., 1994, in: The Impact of Long-Term Monitoring on Variable Star Research, Sterken C. and deGroot M. (eds.), NATO ASI C436. Kluwer, p. 213

Szabados L., 1995, in: Astrophysical Application of Stellar Pulsation, Proc. IAU Coll., No. 155, Stobie R.S., Whitelock P. (eds.), ASP Conf. Ser. 83, 357
Szabados L., 1996, A\&A 311, 189

Szabados L., 1998 (in preparation)

Takase B., 1969, Tokyo Astr. Bul., 2nd Ser., No. 191

Tsessevich V.P., 1952, Odessa Izv. 2, No. 2, 98

Vinkó J., 1991, Ap\&SS 183, 17

Vinkó J., Evans N.R., Kiss L.L., Szabados L., 1998, MNRAS (in press)

Walraven Th., Mueller A.B., Oosterhoff P.Th., 1958, BAN 14, 81

Weaver H.F., Steinmetz D., Mitchell R.I., 1960, Lowell Obs. Bul. 5, 30

Williams A.S., 1918, MNRAS 78, 483

Willson L.A., 1986, in: The Study of Variable Stars Using Small Telescopes; Percy J.R. (ed.). Cambridge Univ. Press, p. 219

Yakimov V.S., 1962, Perem. Zv. 14, 63 